2 Research Square
Preprints are preliminary reports that have not undergone peer review.

They should not be considered conclusive, used to inform clinical practice, or referenced by the media as validated information.

\title{
Socio-demographic characteristics, lifestyles, social support quality and mental health in college students: A cross-sectional study
}

Chao Wang ( $\nabla$ wangch@whu.edu.cn)

Wuhan University

Shijiao Yan

Hainan Medical University

Heng Jiang

La Trobe University

Yingying Guo

Tongji Hospital

Chuanzhu Lv

the Second Affiliated Hospital of Hainan Medical University

Zuxun Lu

Huazhong University of Science and Technology

Yong Gan

Huazhong University of Science and Technology

Research Article

Keywords: China, College students, Influencing factors, Mental health problem, Social support

Posted Date: February 18th, 2022

DOI: https://doi.org/10.21203/rs.3.rs-75584/v2

License: (c) (i) This work is licensed under a Creative Commons Attribution 4.0 International License. Read Full License 


\section{Abstract}

Background: Mental health problem is an important public health issue among college students and is associated with various soical factors. However, these influencing factors were scarcely summarized in Chinese college students comprehensively. This study aims to assess the associations between sociodemographic characteristics, lifestyles, social support quality (SSQ) and mental health among Chinese college students.

Methods: A cross-sectional study was conducted in Wuhan, China, from October 2017 to February 2018. College students from 18 colleges or universities were randomly recruited using multi-stage cluster sampling method. The Multidimensional Scale of Perceived Social Support scale and 12-items General Health Questionnaire were used to estimate students' SSQ and mental health status, respectively. Logistic regression analysis was used to evaluate the associations between socio-demographic characteristics, lifestyles, SSQ and mental health problems.

Results: A total of 10676 (96.24\%) college students were included in the study. Among them, $21.4 \%$ were identified as having mental health problems. Students being a female, aged 18-22 years old, whose mother held college degrees and above, and drinking alcohol were more likely to have mental problems $(P<0.05)$. Contrarily, having general or higher family economic levels, working and resting regularly, and sleeping $\geq 7$ hours could be preventive factors $(P$ $<0.05)$. Especially, there was a decreasing trend in the risk of mental health problems with the improvement of SSQ.

Conclusion: Besides socio-economic and lifestyle factors, social support is a critical factor for mental health among college students. Improving SSQ, especially which from the family, could be an effective method to prevent mental problems among college students.

\section{Background}

Mental health problem is a significant and growing public health issue, its high prevalence and heavy burden have aroused people's attention. A systematic review based on 174 studies across 63 countries showed that the 12-month common mental disorder prevalence was $17.6 \%$ and the lifetime prevalence was $29.2 \%$ [1]. Unfortunately, the effect of mental health problem can be long-lasting or recurrent. Student's mental health is an important topic throughout the education system, which not only affects students' academic performance, but is a significant predictor of personal development [2]. Previous studies on students' mental health problems mostly focused on the primary and secondary school years [3, 4]. However, it is also a prominent health problem among college students, and the depression and anxiety are among the most common [5].

Most of college students are just entering adulthood, it is a crucial time for personal identity development and psychology transition. In this period, they are generally sensitive to the shift of surroundings, such as changes of living and learning environments [6, 7]. On the other hand, entering college/university is generally followed by considerable academic pressure and more adult-like responsibilities, but they have no enough cognitive maturity or foundational skills required for adulthood [8]. A mental health survey performed by WHO in 21 countries showed that $20.3 \%$ of college students had suffered from mental health problems, but only $16.4 \%$ of them received appropriate healthcare [9]. China has the largest number of college students in the world and mental health problem is a prominent health challenge for them, $16 \%-30 \%$ college students have suffered from depression, anxiety, or other mental problems [10]. Some studies also suggested the average prevalence of depression in college students was $30.39 \%$ [11], and the prevalence of anxiety was around $40 \%$ for male and even $45 \%$ for female students [12].

Mental health status is affected by complex reasons, such as socio-demographic characteristics [13, 14], lifestyles [15, 16], as well as family conditions and social networks $[17,18]$. Social support refers to the help provided by individuals who comprise the social network of a person who occupies the position of ego in this network [19], its quality may vary due to the number, intensity and frequency of social contacts. Social support quality is considered as another critical influencing factor for mental health status $[20,21]$, and dissatisfaction with insufficient or poor-quality social supports is closely associated with mental problems in emerging adulthood [19].

Although there were some attempts to estimate the prevalence and influencing factors of mental health status among college students and provided general knowledge of their relationship, the sample sizes of these studies were relatively small [22, 23], or only focused on a specific dimension rather than comprehensive studies. In addition, we have two hypotheses: firstly, there would be a significant trend in relationships between mental health status and SSQ from various resources; secondly, SSQ from the family would be better in preventing mental health problems than those from the friends or significant others. Therefore, we conducted a large-scale epidemiological study among Chinese college students with two objectives. Firstly, we aimed to analyze the influencing factors of mental health problems among Chinese college students; secondly, we sought to evaluate the association between college students' mental health status and SSQ from different sources.

\section{Methods}

Sample and data collection

We conducted a large population-based, cross-sectional study among 18 colleges/universities in Wuhan, Central China, from October 2017 to February 2018. A multi-stage cluster random sampling method was applied in this survey. Firstly, according to subject settings, we categorized the 18 colleges/universities into seven groups: five comprehensive universities, seven universities of science and technology, two universities of finance and economics, and one university of teacher-training, agronomy, nationalities as well as sports, respectively. Secondly, we randomly selected, in the proprotion of students sizes, several classes from each grade (from undergraduate to doctoral degree) in every college/university. Then, all students in selected classes were encouraged to participate in this survey with the voluntary principle and ensured no less than 500 questionnaires were received from each college/university. All participated students were asked to fulfill an online questionnaire on their computers or cellphones. The questionnaire was used to collect students' information including sociodemographic characteristics, individual lifestyles or behaviors, perceived social support, physical and mental health status. Ultimately, a total of 11750 college 
students participated in the survey and 11093 questionnaires were collected on a computer terminal, with a response rate of $94.41 \%$. After excluding those completed in less than five minutes, 10676 qualified questionnaires were included in final statistical analyses, yielding a $96.24 \%$ qualification rate.

This study was approved by the ethics committee of Tongji Medical College institutional review board, Huazhong University of Science and Technology, Wuhan, China. All participants signed informed consent before filling out the questionnaire.

Social support

Multidimensional Scale of Perceived Social Support (MSPSS) [24] consists of 12 items with response options scoring from 1 (very strongly disagree) to 7 (very strongly agree). It estimates SSQ from three sources: family (item 3, 4, 8, and 11), friends (item 6, 7, 9, and 12) and significant others (item 1, 2, 5, and 10) [25]. Scores of all items are added up and then divided by 12. The mean scores ranging from 1 to $2.99,3$ to 5 and 5.01 to 7 are classified as low, medium, and high perceived support levels, respectively [24]. MSPSS has a sound factorial validity (with Cronbach's alpha coefficients of 0.953), and internal consistencies for the full scale and subscales are both satisfactory [26]. The Chinese version has been suggested as a reliable tool for assessing SSQ [27].

Mental health status

The 12-items General Health Questionnaire (GHQ-12) [28] is one of the most widely used versions measuring individual mental health problems. It includes 12 items corresponding to three dimensions: anxiety/depression (item 1, 2, 7, and 10), social dysfunction (item 3, 4, 5, 6, 8, and 9) and deficiency of confidence (item 11 and 12) [29]. There are four answers ranging from "better/healthier than normal" to "much worse/more than usual". The GHQ scoring method (the four options were scored by 0-0-1-1, respectively) has been adopted in our study [30]. Higher score corresponds to worse mental health status. A total score of 4 or more was classified as having notable mental problem [31]. GHQ-12 had satisfactory reliability (with Cronbach's alpha coefficients of 0.886 ) and extensive sensitivity, which had been previously validated.

Socio-demographic characteristics and lifestyles

The questionnaire includes the following demographic variables: age, gender, ethnicity, religious belief, place of residence, single-parent/-child family or not, paternal/maternal education level, and family economic status. Family economic status was assessed by asking the question of "what do you think of your family economic condition?" with optional responses of "very affluent", "more affluent", "the general", "less affluent", or "non-affluent". Based on the responses, family economic status was categorized as good, general, and poor.

Lifestyle variables refer to physical exercise, regular work-rest or not, sleep duration, smoking and alcohol drinking status. Physical exercise was judged from the question of "do you have chronic aerobic exercise (e.g. setting-up exercise, jogging, walking) for 30 minutes and longer three times a week?", and the responses were classified into "never/seldom", "somtimes", and "usually/always". Regular work-rest was estimated by the question of "do you have a regular daily routine?", and the options were also classified into three categories: "never/seldom", "somtimes", and "usually/always". Sleep duration was divided into " $<7$ hours", "7-8.9 hours", and " $\geq 9$ hours" based participants' answers to "In recent three monthes, you sleep for XX hours, XX minutes every day on average." Smoking and alcohol drinking were dichotomized as "yes" and "no" according to participants' responses.

\section{Data Analysis}

Data analyses were performed using the SPSS software (Version 22 for Windows, SPSS Inc, Chicago, IL, U.S.A.). Descriptive analyses included means (standard deviations [SDs]) for continuous variables and frequencies and percentages for categorical data. We analyzed respondents' demographic characteristics, and compared the differences of SSQ and mental health statuses among various demographics by $\chi^{2}$ tests. Potential influencing factors of psychological problem were identified via multivariate logistic regression analyses. Furthermore, we described the correlation between MSPSS and GHQ-12 by matrix analysis and estimated relationships between SSQ and mental problems under different adjustments using trend analysis. Potential confounders included age, gender, ethnicity, religious belief, residence area, single-parent/-child family, parental education level, family economic status, work-rest routine, sleep duration, alcohol drinking, and smoking. Significance level was accepted as $P<0.05$ (two-tailed) for all tests.

\section{Results}

Refering to Table 1, among the included 10676 college students (with a mean age of 19.66 [SD=2.22]), 56.7\% were female, and 2284 (21.4\%) students had mental health problems. The results of $\chi^{2}$ tests suggested that SSQ was associated with gender, age, religious belief, residence area, from single-parent or child family or not, paternal or maternal education level, and family economic status $(P<0.05)$. A lower SSQ (score $1-2.9)$ were found in males, students aged 18-22 years old, and those having religious beliefs, living in rural areas, from single-parent, or non-single-child families. It also showed in students whose parents' education levels were primary or below and those from families with lower economic statuses $(P<0.05)$. For mental health problem, gender, age, ethnicity, religious belief, residence area, from single-parent or -child family or not, paternal or maternal education levels, and family economic status were considered as potential relevant factors $(P<0.05)$. Females, minority students, and students aged $18-21$ years old, having religious beliefs, living in rural areas, from single-parent or non-single-child families, whose parents' education levels were primary or below, and from families with poorer economic status were more likely to have mental problems $(P<0.05)$. 
Table 1

Demographic Characteristics of study participants by perceived social support quality and mental health status.

\begin{tabular}{|c|c|c|c|c|c|c|c|c|c|c|c|}
\hline \multirow[t]{2}{*}{ Demographics } & & \multirow[t]{2}{*}{$\begin{array}{l}\text { Sample } \\
\text { size (\%) }\end{array}$} & \multicolumn{3}{|c|}{ Social support quality } & \multirow[t]{2}{*}{$x^{2}$} & \multirow[t]{2}{*}{$P$} & \multicolumn{2}{|c|}{$\begin{array}{l}\text { Mental health status } \\
(\%)\end{array}$} & \multirow[t]{2}{*}{$x^{2}$} & \multirow[t]{2}{*}{$P$} \\
\hline & & & Low & Medium & High & & & Normal & Problematic & & \\
\hline \multirow[t]{2}{*}{ Gender } & Male & $\begin{array}{l}4625 \\
(43.3)\end{array}$ & 2.2 & 40.2 & 57.6 & 51.547 & $<0.001$ & 80.8 & 19.2 & 22.895 & $<0.001$ \\
\hline & Female & $\begin{array}{l}6051 \\
(56.7)\end{array}$ & 1.3 & 34.5 & 64.2 & & & 76.9 & 23.1 & & \\
\hline \multirow[t]{4}{*}{ Age (years old) } & $<18$ & $680(6.4)$ & 1.8 & 36.9 & 61.3 & 26.571 & $<0.001$ & 81.8 & 18.2 & 12.383 & 0.006 \\
\hline & $18-22$ & $\begin{array}{l}8136 \\
(76.3)\end{array}$ & 1.7 & 38.1 & 60.2 & & & 77.9 & 22.1 & & \\
\hline & $22-26$ & $\begin{array}{l}1640 \\
(15.4)\end{array}$ & 1.6 & 32.3 & 66.0 & & & 80.1 & 19.9 & & \\
\hline & $\geq 26$ & $157(1.9)$ & 2.5 & 28.6 & 69.0 & & & 84.2 & 15.8 & & \\
\hline \multirow[t]{2}{*}{ Ethnicity } & Han & $\begin{array}{l}9476 \\
(88.8)\end{array}$ & 1.7 & 36.9 & 61.4 & 0.110 & 0.946 & 79.1 & 20.9 & 11.955 & 0.001 \\
\hline & Minority & $\begin{array}{l}1200 \\
(11.2)\end{array}$ & 1.8 & 37.3 & 60.9 & & & 74.8 & 25.3 & & \\
\hline \multirow[t]{2}{*}{ Religious belief } & No & $\begin{array}{l}10097 \\
(94.6)\end{array}$ & 1.6 & 36.7 & 61.7 & 14.614 & 0.001 & 78.9 & 21.1 & 9.215 & 0.002 \\
\hline & Yes & $579(5.4)$ & 2.9 & 42.3 & 54.7 & & & 73.6 & 26.4 & & \\
\hline \multirow[t]{2}{*}{ Residence area } & Rural & $\begin{array}{l}4978 \\
(46.6)\end{array}$ & 1.9 & 40.4 & 57.7 & 52.118 & $<0.001$ & 77.7 & 22.3 & 4.740 & 0.029 \\
\hline & Urban & $\begin{array}{l}5698 \\
(53.4)\end{array}$ & 1.5 & 33.9 & 64.5 & & & 79.4 & 20.6 & & \\
\hline \multirow{2}{*}{$\begin{array}{l}\text { Single-parent } \\
\text { family or not }\end{array}$} & Yes & $817(7.7)$ & 3.2 & 44.2 & 52.6 & 34.920 & $<0.001$ & 73.2 & 26.8 & 15.407 & $<0.001$ \\
\hline & No & $\begin{array}{l}9859 \\
(92.3)\end{array}$ & 1.6 & 36.4 & 62.1 & & & 79.1 & 20.9 & & \\
\hline \multirow[t]{2}{*}{$\begin{array}{l}\text { Single-child } \\
\text { family or not }\end{array}$} & Yes & $\begin{array}{l}4931 \\
(46.2)\end{array}$ & 1.7 & 33.9 & 64.4 & 37.386 & $<0.001$ & 79.9 & 20.1 & 9.740 & 0.002 \\
\hline & No & $\begin{array}{l}5745 \\
(53.8)\end{array}$ & 1.7 & 39.6 & 58.7 & & & 77.5 & 22.5 & & \\
\hline \multirow[t]{4}{*}{$\begin{array}{l}\text { Paternal } \\
\text { education level }\end{array}$} & $\begin{array}{l}\text { Elementary school } \\
\text { and below }\end{array}$ & $\begin{array}{l}1558 \\
(14.6)\end{array}$ & 2.2 & 42.6 & 55.2 & 83.970 & $<0.001$ & 76.4 & 23.6 & 9.173 & 0.027 \\
\hline & Junior high school & $\begin{array}{l}3872 \\
(36.3)\end{array}$ & 1.6 & 39.8 & 58.6 & & & 78.7 & 21.3 & & \\
\hline & $\begin{array}{l}\text { High/Secondary } \\
\text { school }\end{array}$ & $\begin{array}{l}2739 \\
(25.7)\end{array}$ & 1.6 & 35.5 & 62.9 & & & 80.2 & 19.8 & & \\
\hline & College and above & $\begin{array}{l}2507 \\
(23.5)\end{array}$ & 1.6 & 30.8 & 67.7 & & & 78.1 & 21.9 & & \\
\hline \multirow[t]{4}{*}{$\begin{array}{l}\text { Matemal } \\
\text { education level }\end{array}$} & $\begin{array}{l}\text { Elementary school } \\
\text { and below }\end{array}$ & $\begin{array}{l}2742 \\
(25.7)\end{array}$ & 2.0 & 42.2 & 55.8 & 95.481 & $<0.001$ & 76.6 & 23.4 & 15.565 & 0.001 \\
\hline & Junior high school & $\begin{array}{l}3711 \\
(34.8)\end{array}$ & 1.5 & 38.6 & 59.8 & & & 79.9 & 20.1 & & \\
\hline & $\begin{array}{l}\text { High/Secondary } \\
\text { school }\end{array}$ & $\begin{array}{l}2395 \\
(22.4)\end{array}$ & 1.5 & 34.4 & 64.1 & & & 80.0 & 20.0 & & \\
\hline & College and above & $\begin{array}{l}1828 \\
(17.1)\end{array}$ & 1.9 & 29.2 & 69.0 & & & 77.1 & 22.9 & & \\
\hline \multirow[t]{3}{*}{$\begin{array}{l}\text { Family economic } \\
\text { status }\end{array}$} & Poor & $\begin{array}{l}3358 \\
(31.5)\end{array}$ & 2.5 & 44.5 & 53.1 & 202.644 & $<0.001$ & 73.8 & 26.2 & 66.843 & $<0.001$ \\
\hline & General & $\begin{array}{l}6373 \\
(59.7)\end{array}$ & 1.3 & 35.2 & 63.5 & & & 80.7 & 19.3 & & \\
\hline & Good & $945(8.9)$ & 1.7 & 22.5 & 75.8 & & & 81.4 & 18.6 & & \\
\hline
\end{tabular}




\begin{tabular}{|c|c|c|c|c|c|c|c|c|c|c|}
\hline \multirow[t]{2}{*}{ Demographics } & \multirow[t]{2}{*}{$\begin{array}{l}\text { Sample } \\
\text { size (\%) }\end{array}$} & \multicolumn{3}{|c|}{ Social support quality } & \multirow[t]{2}{*}{$x^{2}$} & \multirow[t]{2}{*}{$P$} & \multicolumn{2}{|c|}{$\begin{array}{l}\text { Mental health status } \\
(\%)\end{array}$} & \multirow[t]{2}{*}{$x^{2}$} & \multirow[t]{2}{*}{$P$} \\
\hline & & Low & Medium & High & & & Normal & Problematic & & \\
\hline \multirow[t]{2}{*}{ Total } & \multirow{2}{*}{$\begin{array}{l}10676 \\
(100.0)\end{array}$} & 181 & \multirow{2}{*}{$\begin{array}{l}3947 \\
(37.0)\end{array}$} & 6548 & & & 78.6 & 21.4 & & \\
\hline & & $(1.7)$ & & $(61.3)$ & & & (8392) & $(2284)$ & & \\
\hline
\end{tabular}

As shown in Table 2, regression analysis indicated that gender, age, maternal education level, family economic status, work-rest routine, sleep duration, alcohol drinking and SSQ had potential influences on students' psychological status $(P<0.05)$. Among them, female, aged $18-21$ years old, maternal education level was college and above, drinking alcohol were independently associated with poorer mental health statuses (odds ratios (ORs) were between 1.245 and 1.362 , all $P<0.05$ ). While middle and higher family economic status, regular work-rest routine, sleep duration $\geq 7$ hours, having moderate and high social support quality were more likely to have better mental health statuses (ORs ranged from 0.245 to 0.709 , all $P<0.05$ ). Among them, SSQ was one of the most significant influencing factors for students' mental health problems (moderate vs. low: OR=0.521, 95\% $\mathrm{Cl}=0.383-0.708$; high vs. low: OR=0.245, 95\% $\mathrm{Cl}=0.180$ 0.333).

Table 2

Multivariate logistic regression analyses for the influencing factors of mental health among college students.

\begin{tabular}{|c|c|c|c|c|c|c|c|c|}
\hline \multirow[t]{2}{*}{ Variables } & & \multirow[t]{2}{*}{$\beta$} & \multirow[t]{2}{*}{ S.E. } & \multirow[t]{2}{*}{ Wald $\chi^{2}$} & \multirow[t]{2}{*}{$O R$} & \multicolumn{2}{|c|}{$95 \% \mathrm{Cl}$} & \multirow[t]{2}{*}{$P$} \\
\hline & & & & & & LL & UL & \\
\hline Gender (ref=male) & Female & 0.309 & 0.055 & 31.581 & 1.362 & 1.223 & 1.517 & $<0.001$ \\
\hline \multirow[t]{3}{*}{ Age (years old) $($ ref $=<18)$} & $18-22$ & 0.269 & 0.107 & 6.334 & 1.308 & 1.061 & 1.613 & 0.012 \\
\hline & $23-26$ & 0.230 & 0.122 & 3.552 & 1.258 & 0.991 & 1.597 & 0.059 \\
\hline & $\geq 26$ & 0.012 & 0.225 & 0.003 & 1.012 & 0.651 & 1.572 & 0.958 \\
\hline \multirow{3}{*}{$\begin{array}{l}\text { Maternal education level } \\
\text { (ref=Elementary school and below) }\end{array}$} & Junior high school & -0.094 & 0.067 & 1.973 & 0.910 & 0.798 & 1.038 & 0.160 \\
\hline & High/Secondary school & -0.001 & 0.086 & 0.000 & 0.999 & 0.844 & 1.183 & 0.989 \\
\hline & College and above & 0.219 & 0.108 & 4.097 & 1.245 & 1.007 & 1.540 & 0.043 \\
\hline \multirow{2}{*}{$\begin{array}{l}\text { Family economic status } \\
\text { (ref=Lower) }\end{array}$} & General & -0.344 & 0.058 & 35.042 & 0.709 & 0.633 & 0.794 & $<0.001$ \\
\hline & Higher & -0.369 & 0.108 & 11.807 & 0.691 & 0.560 & 0.853 & $<0.001$ \\
\hline \multirow{2}{*}{$\begin{array}{l}\text { Work-rest routine } \\
\text { (ref=Never/Seldom) }\end{array}$} & Sometimes & -0.266 & 0.065 & 16.493 & 0.767 & 0.674 & 0.872 & $<0.001$ \\
\hline & Usually/Always & -0.613 & 0.062 & 96.318 & 0.542 & 0.479 & 0.612 & $<0.001$ \\
\hline \multirow[t]{2}{*}{ Sleep duration (hours) (ref $=<7$ ) } & $7-9$ & -0.352 & 0.054 & 43.138 & 0.704 & 0.633 & 0.781 & $<0.001$ \\
\hline & $\geq 9$ & -0.347 & 0.129 & 7.286 & 0.707 & 0.549 & 0.909 & 0.007 \\
\hline Alcohol drinking (ref=No) & Yes & 0.249 & 0.062 & 15.924 & 1.283 & 1.135 & 1.450 & $<0.001$ \\
\hline \multirow[t]{2}{*}{ Social support quality (ref=Low level) } & Moderate level & -0.653 & 0.157 & 17.240 & 0.521 & 0.383 & 0.708 & $<0.001$ \\
\hline & High level & -1.407 & 0.157 & 79.878 & 0.245 & 0.180 & 0.333 & $<0.001$ \\
\hline
\end{tabular}

In Table 3, the correlation matrix suggested that SSQ was negatively associated with mental problem ( $r=-0.184)$. Among the three social support sources, family provided the strongest effect on mental health problem ( $r=-0.182)$, then followed by friends ( $r=-0.167)$ and significant others $(r=-0.157)$. Within the MSPSS, the family, friends and significant others subscales were highly correlated with each other ( $r$ between 0.586 and 0.717$)$ and with the overall scale $(r$ between 0.787 and 0.835 ). 
Table 3

Correlation between MSPSS and GHQ-12.

\begin{tabular}{|c|c|c|c|c|c|}
\hline Variables & Quality of SSQ & Subscale-family & Subscale-friends & Subscale-significant others & Mental health problem \\
\hline Quality of SSQ & 1.000 & & & & \\
\hline Subscale-family & 0.787 & 1.000 & & & \\
\hline Subscale-friends & 0.789 & 0.586 & 1.000 & & \\
\hline Subscale-significant others & 0.835 & 0.656 & 0.717 & 1.000 & \\
\hline Mental health problem & -0.184 & -0.182 & -0.167 & -0.157 & 1.000 \\
\hline
\end{tabular}

As presented in Table 4, compared with low SSQ, both moderate and high SSQ reduced the risk of mental health problem (ORs ranged from 0.184 to $0.526, P$ $<0.05)$. In different models of each subscale, there were significant difference in effect of different SSQ on mental health status $\left(P_{\text {difference }}\right.$ all $\left.<0.05\right)$.

Furthermore, in all three models with different adjustments, there were significant positive trends in associations between both full scale and subscales of SSQ and mental health problems ( $P_{\text {trend }}$ all $\left.<0.001\right)$. Especially, in model 3, with the full adjustment, both higher and moderate SSQ had greater negative impacts on mental problems than the low SSQ $(\mathrm{OR}=0.249,95 \% \mathrm{Cl}=0.183-0.338 ; \mathrm{OR}=0.526,95 \% \mathrm{Cl}=0.386-0.715$, respectively). In subscales, family support had the strongest preventive effect on students' mental health problems (high/moderate SSQ $=0.412 / 0.219)$, then followed by friends supports $(0.434 / 0.234)$ and significant others supports $(0.524 / 0.285)$.

Table 4

Multivariate logistic regression analyses for the association between social support quality and mental health status.

\begin{tabular}{|c|c|c|c|c|c|c|c|c|c|c|c|c|c|c|c|}
\hline \multirow{3}{*}{$\begin{array}{l}\text { Variables } \\
\text { OR }\end{array}$} & \multicolumn{4}{|c|}{ Unadjusted } & \multicolumn{4}{|c|}{ Model $1^{a}$} & \multicolumn{4}{|c|}{ Model $2^{b}$} & \multicolumn{3}{|c|}{ Model $3^{c}$} \\
\hline & \multirow[t]{2}{*}{$95 \% \mathrm{Cl}$} & \multirow[b]{2}{*}{ LL } & \multirow{2}{*}{$\begin{array}{l}\mathrm{P} \\
\text { trend }\end{array}$} & \multirow[t]{2}{*}{ OR } & \multicolumn{2}{|l|}{$95 \% \mathrm{Cl}$} & \multirow{2}{*}{$\begin{array}{l}P \\
\text { trend }\end{array}$} & \multirow[t]{2}{*}{ OR } & \multicolumn{2}{|l|}{$95 \% \mathrm{Cl}$} & \multirow{2}{*}{$\begin{array}{l}P \\
\text { trend }\end{array}$} & \multirow[t]{2}{*}{ OR } & \multicolumn{2}{|l|}{$95 \% \mathrm{Cl}$} & \multirow{2}{*}{$\begin{array}{l}P \\
\text { trend }\end{array}$} \\
\hline & & & & & UL & LL & & & UL & LL & & & UL & LL & \\
\hline \multirow{2}{*}{$\begin{array}{l}\text { SSQ } \\
\text { (ref.=Low) }\end{array}$} & Medium & 0.489 & 0.363 & 0.661 & $<0.001$ & 0.472 & 0.349 & 0.637 & $<0.001$ & 0.496 & 0.366 & 0.672 & $<0.001$ & 0.526 & 0.386 \\
\hline & High & 0.214 & 0.159 & 0.289 & & 0.203 & 0.150 & 0.274 & & 0.219 & 0.162 & 0.297 & & 0.249 & 0.183 \\
\hline$P_{\text {difference }}$ & & & $<0.05$ & & & & $<0.05$ & & & & $<0.05$ & & & & $<0.05$ \\
\hline \multirow{2}{*}{$\begin{array}{l}\text { SSQ- } \\
\text { Family } \\
\text { (ref.=Low) }\end{array}$} & Medium & 0.385 & 0.303 & 0.489 & $<0.001$ & 0.382 & 0.300 & 0.485 & $<0.001$ & 0.403 & 0.317 & 0.513 & $<0.001$ & 0.412 & 0.323 \\
\hline & High & 0.187 & 0.147 & 0.237 & & 0.184 & 0.145 & 0.233 & & 0.199 & 0.156 & 0.254 & & 0.219 & 0.171 \\
\hline$P$ difference & & & $<0.05$ & & & & $<0.05$ & & & & $<0.05$ & & & & $<0.05$ \\
\hline \multirow{2}{*}{$\begin{array}{l}\text { SSQ- } \\
\text { Friends } \\
\text { (ref.=Low) }\end{array}$} & Medium & 0.400 & 0.308 & 0.521 & $<0.001$ & 0.393 & 0.302 & 0.511 & $<0.001$ & 0.415 & 0.318 & 0.542 & $<0.001$ & 0.434 & 0.332 \\
\hline & High & 0.202 & 0.156 & 0.263 & & 0.196 & 0.151 & 0.255 & & 0.211 & 0.161 & 0.275 & & 0.234 & 0.179 \\
\hline$P$ difference & & & $<0.05$ & & & & $<0.05$ & & & & $<0.05$ & & & & $<0.05$ \\
\hline \multirow{2}{*}{$\begin{array}{l}\text { SSQ- } \\
\text { Significant } \\
\text { others } \\
\text { (ref.=Low) }\end{array}$} & Medium & 0.504 & 0.384 & 0.662 & $<0.001$ & 0.482 & 0.366 & 0.635 & $<0.001$ & 0.502 & 0.380 & 0.661 & $<0.001$ & 0.524 & 0.396 \\
\hline & High & 0.256 & 0.195 & 0.335 & & 0.237 & 0.180 & 0.311 & & 0.253 & 0.192 & 0.334 & & 0.285 & 0.215 \\
\hline$P_{\text {difference }}$ & & & $<0.05$ & & & & $<0.05$ & & & & $<0.05$ & & & & $<0.05$ \\
\hline
\end{tabular}

Note, LL= Low limit; UL=Upper limit; SSQ=Social support quality, ref.=reference.

${ }^{\text {a }}$ Adjusted for age and gender.

${ }^{b}$ Furthermore adjusted for ethnicity, religious belief, residence area, single-parent or not, signal-child or not, family economic status, paternal and maternal ed

${ }^{\mathrm{c}}$ Furthermore adjusted for exercise, life schedule and sleep duration.

\section{Discussion}


A total of 10676 college/university students were included in this study and the prevalence of mental health problem was $21.4 \%$. Although this result was lower than the finding (28.0\%) from a survey conducted in Finnish university students [32], it was higher than results of a cross-nation mental health survey (20.3\%) [9] and another one (19.0\%) from Hungary [33], all above studies were conducted among college students using the GHQ-12. These differences might be attributed to racial, cultural, and socio-demographic disparities [34,35]. With the increase of SSQ, the risk of mental health problem among college students showed a significant decreasing trend, suggesting that improving the SSQ could be an effective and practical method to prevent mental health problems of college students.

Compared with liberal education in western developed countries, China follows a relatively conservative education model. Under this model, schools are prone to pursue attractive acdemic achievement rather than students' quality-oriented education and healthy psychological development [36]. Heavy academic burden and insufficient healthcares on mental health lead to negative mental health status among Chinese stduents. However, because mental health problems are long-lasting throughout the entire education period, plus the transitional period of psychological development, which is sensitive to surrounding environment, lifestyles or behaviors, and social supports from family, friends or significant others, the mental health problem are prominent among college students.

Mental health problem was associated with various factors. Similar to previous studies [23], the female generally lived with more delicate emotions and were prone to have negative views of their health than males, they were more likely to have mental problems. Compared with the older college students, the freshmen, mostly aged 18-21, faced confused lifetime planning, cash-strapped living, and less social experience, which made them anxious and stressful. Mother played a pivotal role in the development of children's mental health [37, 38], but we found that mother with college degree or above was a risk factor for students' mental health. A possible explanation is higher academic degree generally corresponds to longer work time and heavier work loads, which deprives the time and energy to accompany with children. Alcohol drinking was negatively associated with mental health. Students who drank alcohol tended to have decreased sensitivity, intense emotions, and interpersonal conflicts, and eventually increased the risk of mental health problems [39, 40]. Nevertheless, the function between alcohol drinking and mental problem could be bi-directional, college students also opted to cope with their depression, stress and other mental illnesses by drinking. This bidirectional relationship warrants further studies.

Higher family economic status could protect students from being mental problems, which aligned with the previous evidences [41]. Coupled with the increasing self-esteem of college students, the gap between subjective demands and objective facts due to poor economic condition contributed to psychological imbalance and ultimately induced mental problems [42]. Students followed regular work-rest schedule had significant lower risk of mental problems than those who never/seldom work-rest regularly. This finding was partly supported by a study based on the UK Bio-bank, where circadian disruption was reliably associated with various adverse mental health outcomes [43]. However, as its study sample aged 37-73 years old, the result might be not applicable for college students, further prospective studies and randomized controlled trials are needed to confirm such relationship. In addition, sleep seven hours and longer would decline the risk of mental health problem significantly, which was consistent with results of a recent meta-analysis [44]. Adequate sleep could preserve the homeostasis of affective brain, and optimally prepare next-day emotional functioning, leading to a stable and healthy mental status [45].

Compared with low SSQ, moderate and high level of SSQ were protective factors for students' mental health. From the correlation coefficient matrix analysis, we found that SSQ and its subscales were negatively correlated with mental health problem, which were consistent with previous studies [46]. Under different levels of adjustment, medium and high SSQ and their subscales were all negatively associated with mental problem when compared to low SSQ. Adequate high-quality social supports could give individuals comfortable mental consolation and a sense of security, which benefits college students to keep a healthy psychological status [47]. In general, family is the most important source of social support. Due to the conservative family values and collectivist nature of Chinese society, family ties were deemed as the most important social relationships [48]. People affiliated with mental health problems often create the feelings of stigma or shame. In this case, the family could play a key role in guiding family members with psychological problems to receive treatment, and making patients healthy through active interventions [49]. However, several studies listed friends as the most important source of supports, ahead of family and significant others [50]. The reason might be that most college students lived with friends in the campus rather than their family members, friends could timely find psychological change of each other and social supports from friends could offer sufficient mental assistance. However, in this study, SSQ could improve college students' mental health, regardless of from family, friends or significant others. This finding informed that university administrators and teachers should improve students' SSQ, especially the supports from family and friends, so as to prevent mental problems in college students better.

\section{Strengths And Limitations}

There were several strengths in this study. Firstly, we included 10676 students in this study via the multi-stage random cluster sampling. It was a big sample size among relevant studies, guaranteeing the results were credible to some extent. Secondly, we obtained some important findings. For example, there was a decreasing trend of being mental problem with the improvement of SSQ. This result further supplemented and confirmed the influencing factor network of college students' mental health, and provided statistical data for international comparisons on this topic.

However, some limitations should be acknowledged. Firstly, the study was a cross-sectional design, the results only suggested the observational correlation rather than the causality between SSQ and mental health problems. Secondly, students' SSQ and mental health statuses were collected through self-reported, which might be a potential source of information bias. However, as the questionnaire was filled out in anonymous forms, obtained data might be objective. Thirdly, the classification on socio-demographic and lifestyle variables (e.g. alcohol drinking, family economic status) in this study were simple, which, to some extent, limited further analyses on the impact of specific socio-demographic characteristics or lifestyles on individual mental health status. Studies based on detailed classification on socio-demographic and lifestyle variables should be conducted in the future. Finally, this study was conducted in Wuhan city, which might limit the generality to other regions. However, college students in Wuhan were from across the country, which could make up for the lack of sample representation to some extent. 


\section{Conclusion}

Besides socio-economic and lifestyle factors, social support is a positive and critical factor for mental health. The higher SSQ, especially which from the family, could be better in preventing mental health problems those from friends or significant others. Our findings may provide valuable and practical clues for the prevention of mental disorder among college students.

\section{Abbreviations}

SSQ, social support quality

MSPSS, Multidimensional Scale of Perceived Social Support

GHQ-12, the 12-items General Health Questionnaire

SD, standard deviation

OR, odds ratio

Ref, reference

\section{Declarations}

\section{Ethics approval and consent to participate}

This study was approved by the ethics committee of Tongji Medical College institutional review board, Huazhong University of Science and Technology, Wuhan, China. All participants signed informed consent before filling out the questionnaire. The submission and publication of the manuscript have been approved by all authors and tacitly or explicitly by the responsible authorities of the institutes where the survey was carried out. Human data included in this manuscript was obtained in compliance with the principles outlined in the Helsinki Declaration.

\section{Consent for publication}

Not applicable.

\section{Availability of data and materials}

All data generated or analysed during this study are included in this manuscript. The datasets generated and/or analyzed during the current study are not publicly available due the data is collected by the project team, we can use it with authorization, but cannot share it publicly, but are available from the corresponding author on reasonable request.

\section{Competing interests}

The authors declare that they have no competing interests.

\section{Funding}

This study was supported by the National Social Science Foundation of China (Grant No. 18ZDA085). The funders had no role in study design, data collection and analysis, decision to publish, or preparation of the manuscript.

\section{Author contributions}

CW designed of the questionnaire, participated in the survey and analyzed the data, wrote the draft of the paper. SY and YG designed of the questionnaire, analyzed the data and cross-checked the paper. $\mathrm{HJ}$ and YG checked the draft of the paper and gave advice on statistical methodology. CL contributed to the design of the paper. ZL provided suggestions and supervision of the work. All authors read and approved the final version.

\section{Acknowledgements}

We would like to thank the 11093 study participants in this survey and all staff members involved in this study for their efforts in the data collection. We also want to thank Miss Mia Miler, an assistant researcher from School of Psychology and Public Health, La Trobe University, Australia, for her help in proofreading and editing during the revision of the study.

\section{References}

1. Steel Z, Marnane C, Iranpour C, Chey T, Jackson JW, Patel V, Silove D: The global prevalence of common mental disorders: a systematic review and metaanalysis 1980-2013. International journal of epidemiology 2014, 43(2):476-493.

2. Lefkowitz ES: "Things have gotten better": Developmental changes among emerging adults after the transition to university. J Adolesc Res 2005, 20:4063. 
3. Fenwick-Smith A, Dahlberg EE, Thompson SC: Systematic review of resilience-enhancing, universal, primary school-based mental health promotion programs. BMC psychology 2018, 6(1):30.

4. Werner-Seidler A, Perry Y, Calear AL, Newby JM, Christensen H: School-based depression and anxiety prevention programs for young people: A systematic review and meta-analysis. Clinical psychology review 2017, 51:30-47.

5. Habihirwe P, Porovecchio S, Bramboiu I, Ciobanu E, Croituru C, Cazacu I, Peze T, Ladner J, Tavolacci M: Depression, anxiety and stress among college students in three European countries. European journal of public health 2018, 28(suppl_4).

6. Arnett JJ: Emerging adulthood. A theory of development from the late teens through the twenties. The American psychologist 2000, 55(5):469-480.

7. Arnett JJ, Zukauskiene R, Sugimura K: The new life stage of emerging adulthood at ages 18-29 years: implications for mental health. The lancet Psychiatry 2014, 1(7):569-576.

8. Pedrelli P, Nyer M, Yeung A, Zulauf C, Wilens T: College Students: Mental Health Problems and Treatment Considerations. Academic psychiatry: the journal of the American Association of Directors of Psychiatric Residency Training and the Association for Academic Psychiatry 2015, 39(5):503-511.

9. Auerbach RP, Alonso J, Axinn WG, Cuijpers P, Ebert DD, Green JG, Hwang I, Kessler RC, Liu H, Mortier P et al: Mental disorders among college students in the World Health Organization World Mental Health Surveys. Psychological medicine 2016, 46(14):2955-2970.

10. J L: Overview on study progress of college students' mental health in recent 20 years. Career Horizon 2009(10):168-169.

11. Jiang CX, Li ZZ, Chen P, Chen LZ: Prevalence of Depression Among College-Goers in Mainland China: A Methodical Evaluation and Meta-Analysis. Medicine 2015, 94(50):e2071.

12. Gao W, Ping S, Liu X: Gender differences in depression, anxiety, and stress among college students: A longitudinal study from China. Journal of affective disorders 2020, 263:292-300.

13. Jiang S, Li C, Fang X: Socioeconomic status and children's mental health: Understanding the mediating effect of social relations in Mainland China. Journal of Community Psychology 2018, 46(2):213-223.

14. Zach A, Meyer N, Hendrowarsito L, Kolb S, Bolte G, Nennstiel-Ratzel U, Stilianakis NI, Herr C, Group GMES: Association of sociodemographic and environmental factors with the mental health status among preschool children-Results from a cross-sectional study in Bavaria, Germany. International journal of hygiene and environmental health 2016, 219(4-5):458-467.

15. Nagasu M, Kogi K, Yamamoto I: Association of socioeconomic and lifestyle-related risk factors with mental health conditions: a cross-sectional study. BMC public health 2019, 19(1):1759.

16. Cheah YK, Azahadi M, Phang SN, Abd Manaf NH: Sociodemographic, Lifestyle, and Health Factors Associated With Depression and Generalized Anxiety Disorder Among Malaysian Adults. Journal of primary care \& community health 2020, 11:2150132720921738.

17. Ridout B, Campbell A: The Use of Social Networking Sites in Mental Health Interventions for Young People: Systematic Review. Journal of medical Internet research 2018, 20(12):e12244.

18. Cobham VE, McDermott B, Haslam D, Sanders MR: The Role of Parents, Parenting and the Family Environment in Children's Post-Disaster Mental Health. Current psychiatry reports 2016, 18(6):53.

19. Martinez-Hernaez A, Carceller-Maicas N, DiGiacomo SM, Ariste S: Social support and gender differences in coping with depression among emerging adults: a mixed-methods study. Child and adolescent psychiatry and mental health 2016, 10:2.

20. Tol WA, Barbui C, Galappatti A, Silove D, Betancourt TS, Souza R, Golaz A, van Ommeren M: Mental health and psychosocial support in humanitarian settings: linking practice and research. Lancet (London, England) 2011, 378(9802):1581-1591.

21. Xu Q, Li S, Yang L: Perceived social support and mental health for college students in mainland China: the mediating effects of self-concept. Psychology, health \& medicine 2019, 24(5):595-604.

22. Zhang J, Qi Q, Delprino RP: Psychological health among Chinese college students: a rural/urban comparison. Journal of child and adolescent mental health 2017, 29(2):179-186.

23. Zhang M, Zhang J, Zhang F, Zhang L, Feng D: Prevalence of psychological distress and the effects of resilience and perceived social support among Chinese college students: Does gender make a difference? Psychiatry research 2018, 267:409-413.

24. Gregory D. Zimet NWD, Sara G. Zimet, Gordon K. Farley: The Multidimensional Scale of Perceived Social Support. Journal of personality assessment 1988, 52(1):30-41.

25. Osman A, Lamis DA, Freedenthal S, Gutierrez PM, McNaughton-Cassill M: The multidimensional scale of perceived social support: analyses of internal reliability, measurement invariance, and correlates across gender. Journal of personality assessment 2014, 96(1):103-112.

26. Stewart RC, Umar E, Tomenson B, Creed F: Validation of the multi-dimensional scale of perceived social support (MSPSS) and the relationship between social support, intimate partner violence and antenatal depression in Malawi. BMC psychiatry 2014, 14:180.

27. Guan NC, Seng LH, Hway Ann AY, Hui KO: Factorial validity and reliability of the Malaysian simplified Chinese version of Multidimensional Scale of Perceived Social Support (MSPSS-SCV) among a group of university students. Asia-Pacific journal of public health 2015, 27(2):225-231.

28. Romppel M, Braehler E, Roth M, Glaesmer H: What is the General Health Questionnaire-12 assessing? Dimensionality and psychometric properties of the General Health Questionnaire-12 in a large scale German population sample. Comprehensive psychiatry 2013, 54(4):406-413.

29. Graetz B: Multidimensional properties of the General Health Questionnaire. Social psychiatry and psychiatric epidemiology 1991, 26(3):132-138.

30. General Health Questionnaire (GHQ)

31. Wang S, Li B, Ungvari GS, Ng CH, Chiu HF, Kou C, Liu Y, Tao Y, Wu Y, Fu Y et al: Poor mental health status and its associations with demographic characteristics and chronic diseases in Chinese elderly. Social psychiatry and psychiatric epidemiology 2016, 51(10):1449-1455.

Page $9 / 10$ 
32. Oksanen A, Laimi K, Bjorklund K, Loyttyniemi E, Kunttu K: A 12-year Trend of Psychological Distress: National Study of Finnish University Students. Central European journal of public health 2017, 25(2):113-119.

33. Biro E, Adany R, Kosa K: Mental health and behaviour of students of public health and their correlation with social support: a cross-sectional study. BMC public health 2011, 11:871.

34. Reiss F: Socioeconomic inequalities and mental health problems in children and adolescents: a systematic review. Social science \& medicine 2013 , 90:24-31.

35. Spence ND, Wells S, Graham K, George J: Racial Discrimination, Cultural Resilience, and Stress. Canadian journal of psychiatry Revue canadienne de psychiatrie 2016, 61(5):298-307.

36. Jiang YG: Current Thinking and Liberal Arts Education in China. Ph.D. Ann Arbor: Boston College; 2013.

37. Arroyo-Borrell E, Renart G, Saurina C, Saez M: Influence maternal background has on children's mental health. International journal for equity in health 2017, 16(1):63.

38. Meyrose AK, Klasen F, Otto C, Gniewosz G, Lampert T, Ravens-Sieberer U: Benefits of maternal education for mental health trajectories across childhood and adolescence. Social science \& medicine 2018, 202:170-178.

39. Levin ME, Lillis J, Seeley J, Hayes SC, Pistorello J, Biglan A: Exploring the relationship between experiential avoidance, alcohol use disorders, and alcoholrelated problems among first-year college students. Journal of American college health: J of ACH 2012, 60(6):443-448.

40. Miller JW, Naimi TS, Brewer RD, Jones SE: Binge drinking and associated health risk behaviors among high school students. Pediatrics 2007, 119(1):7685.

41. Gonzales NA, Coxe S, Roosa MW, White RM, Knight GP, Zeiders KH, Saenz D: Economic hardship, neighborhood context, and parenting: prospective effects on Mexican-American adolescent's mental health. American journal of community psychology 2011, 47(1-2):98-113.

42. Wickrama KA, Surjadi FF, Lorenz FO, Conger RD, Walker C: Family Economic Hardship and Progression of Poor Mental Health in Middle-aged Husbands and Wives. Family relations 2012, 61(2):297-312.

43. Lyall LM, Wyse CA, Graham N, Ferguson A, Lyall DM, Cullen B, Celis Morales CA, Biello SM, Mackay D, Ward J et al: Association of disrupted circadian rhythmicity with mood disorders, subjective wellbeing, and cognitive function: a cross-sectional study of 91105 participants from the UK Biobank. The lancet Psychiatry 2018.

44. Baglioni C, Nanovska S, Regen W, Spiegelhalder K, Feige B, Nissen C, Reynolds CF, Riemann D: Sleep and mental disorders: A meta-analysis of polysomnographic research. Psychological bulletin 2016, 142(9):969-990.

45. Goldstein AN, Walker MP: The role of sleep in emotional brain function. Annual review of clinical psychology 2014, 10:679-708.

46. Guan NC, Sulaiman AR, Seng LH, Ann AY, Wahab S, Pillai SK: Factorial validity and reliability of the Tamil version of multidimensional scale of perceived social support among a group of participants in university malaya medical centre, malaysia. Indian journal of psychological medicine 2013, 35(4):385388.

47. Ditzen B, Heinrichs M: Psychobiology of social support: the social dimension of stress buffering. Restorative neurology and neuroscience 2014, 32(1):149-162.

48. Zeng W, North N, Kent B: Family and social aspects associated with depression among older persons in a Chinese context. International journal of older people nursing 2013, 8(4):299-308.

49. Patterson JE, Edwards TM, Vakili S: Global Mental Health: A Call for Increased Awareness and Action for Family Therapists. Family process 2018 , 57(1):70-82.

50. Mahon NE, Yarcheski A: Parent and Friend Social Support and Adolescent Hope. Clinical nursing research 2017, 26(2):224-240. 